\title{
An ordered Turán problem for bipartite graphs
}

\author{
Craig Timmons \\ Department of Mathematics \\ University of California San Diego \\ La Jolla, CA 92093 \\ ctimmons@ucsd.edu
}

Submitted: June 22, 2012; Accepted: Dec 2, 2012; Published: Dec 13, 2012

Mathematics Subject Classification: 05C35

\begin{abstract}
Let $F$ be a graph. A graph $G$ is $F$-free if it does not contain $F$ as a subgraph. The Turán number of $F$, written $\operatorname{ex}(n, F)$, is the maximum number of edges in an $F$-free graph with $n$ vertices. The determination of Turán numbers of bipartite graphs is a challenging and widely investigated problem. In this paper we introduce an ordered version of the Turán problem for bipartite graphs. Let $G$ be a graph with $V(G)=\{1,2, \ldots, n\}$ and view the vertices of $G$ as being ordered in the natural way. A zig-zag $K_{s, t}$, denoted $Z_{s, t}$, is a complete bipartite graph $K_{s, t}$ whose parts $A=\left\{n_{1}<n_{2}<\cdots<n_{s}\right\}$ and $B=\left\{m_{1}<m_{2}<\cdots<m_{t}\right\}$ satisfy the condition $n_{s}<m_{1}$. A zig-zag $C_{2 k}$ is an even cycle $C_{2 k}$ whose vertices in one part precede all of those in the other part. Write $\mathcal{Z}_{2 k}$ for the family of zig-zag $2 k$-cycles. We investigate the Turán numbers $\operatorname{ex}\left(n, Z_{s, t}\right)$ and $\operatorname{ex}\left(n, \mathcal{Z}_{2 k}\right)$. In particular we show $\operatorname{ex}\left(n, Z_{2,2}\right) \leqslant \frac{2}{3} n^{3 / 2}+O\left(n^{5 / 4}\right)$. For infinitely many $n$ we construct a $Z_{2,2}$-free $n$ vertex graph with more than $(n-\sqrt{n}-1)+\operatorname{ex}\left(n, K_{2,2}\right)$ edges.
\end{abstract}

Keywords: Turán problem; bipartite graphs;

\section{Introduction}

Let $\mathcal{F}$ be a family of graphs. A graph $G$ is $\mathcal{F}$-free if $G$ contains no subgraph isomorphic to a graph in $\mathcal{F}$. The Turán number of $\mathcal{F}$ is the maximum number of edges in an $n$ vertex graph that is $\mathcal{F}$-free. Write $\operatorname{ex}(n, \mathcal{F})$ for this maximum and when $\mathcal{F}$ consists of a single graph $F$, write $\operatorname{ex}(n, F)$ instead of $\operatorname{ex}(n,\{F\})$. Turán problems have a rich history in extremal graph theory. While many Turán problems have been solved, there are still many open problems such as determining the Turán number of $C_{8}$, the Turán number of $K_{4,4}$, and the Turán number of the family $\left\{C_{3}, C_{4}\right\}$. The earliest result in this field is 
Mantel's Theorem proved in 1907. Mantel proved $\operatorname{ex}\left(n, K_{3}\right)=\left\lfloor\frac{n}{2}\right\rfloor\left\lceil\frac{n}{2}\right\rceil$ and the $n$-vertex $K_{3}$-free graphs with ex $\left(n, K_{3}\right)$ edges are complete bipartite graphs with part sizes as equal as possible. In 1940 Turán extended Mantel's Theorem and determined the Turán number of $K_{t}, t \geqslant 3$. Turán's Theorem is considered to be the first theorem of extremal graph theory. When $\mathcal{F}$ consists of non-bipartite graphs the Erdős-Stone-Simonovits Theorem determines $\operatorname{ex}(n, \mathcal{F})$ asymptotically.

Theorem 1 (Erdős, Stone, Simonovits). Let $\mathcal{F}$ be a family of graphs and let $r=$ $\min _{F \in \mathcal{F}} \chi(F)$. If $r \geqslant 2$ then

$$
\operatorname{ex}(n, \mathcal{F})=\left(1-\frac{1}{r-1}\right)\left(\begin{array}{l}
n \\
2
\end{array}\right)+o\left(n^{2}\right)
$$

When $\mathcal{F}$ contains bipartite graphs, the Erdős-Stone-Simonovits Theorem gives $\operatorname{ex}(n, \mathcal{F})=o\left(n^{2}\right)$. More precise results can be obtained by using different counting arguments and algebraic constructions.

In this paper we introduce an ordered Turán problem for bipartite graphs. Given an $n$-vertex graph $G$, label its vertices with the numbers $[n]:=\{1,2, \ldots, n\}$ using each number exactly once. This induces a natural ordering of the vertices of $G$ and we use this ordering to distinguish between different types of a fixed subgraph. This idea is not new to Turán theory. Czipszer, Erdős, and Hajnal [8] and Dudek and Rödl [9] investigated Turán problems for increasing paths of length $k$. An increasing path of length $k$ is a sequence of $k$ edges $n_{1} n_{2}, n_{2} n_{3}, \ldots, n_{k} n_{k+1}$ such that $n_{i}<n_{i+1}$ for $1 \leqslant i \leqslant k$.

Let $H$ be a bipartite graph with parts $A$ and $B$. Let $f:\{1,2\} \rightarrow\{A, B\}$ be a bijection and call $f$ an ordering of the parts. A zig-zag $H$ relative to $f$ and bipartition $\{A, B\}$ is a copy of $H$ in $G$ such that all of the vertices in $f(1)$ precede all of the vertices in $f(2)$ in the ordering of $V(G)=[n]$. One of the reason we consider zig-zag complete bipartite graphs as opposed to complete bipartite graphs that do not zig-zag is because there exist graphs with $\frac{1}{8} n^{2}+o\left(n^{2}\right)$ edges that do not contain increasing paths of length 2 . One such graph is obtained by joining each even vertex to all of the odd vertices that come after it in the ordering. If a complete bipartite graph does not zig-zag then it will contain an increasing path of length 2. In contrast, if a zig-zag bipartite graph is forbidden then the number of edges will not be quadratic in $n$. Our focus will be on zig-zag complete bipartite graphs and zig-zag even cycles so we specialize the notation.

As before let $G$ be an $n$-vertex graph with $V(G)=[n]$ and consider the vertices of $G$ as being ordered. A zig-zag $K_{s, t}$, which will be denoted by $Z_{s, t}$, is a $K_{s, t}$ whose parts $A=\left\{n_{1}<n_{2}<\cdots<n_{s}\right\}$ and $B=\left\{m_{1}<m_{2}<\cdots<m_{t}\right\}$ satisfy the condition $n_{s}<m_{1}$. A zig-zag $2 k$-cycle, denoted $Z_{2 k}$, is a $2 k$-cycle whose vertices are $\left\{n_{1}<n_{2}<\cdots<n_{2 k}\right\}$ and $A=\left\{n_{1}, \ldots, n_{k}\right\}, B=\left\{n_{k+1}, \ldots, n_{2 k}\right\}$ is the bipartition. Let $\mathcal{Z}_{2 k}$ be the family of all zig-zag $2 k$-cycles. Observe that for $k=2, \mathcal{Z}_{2 k}$ consists of a single graph and we simply write $Z_{4}$ for this family. 

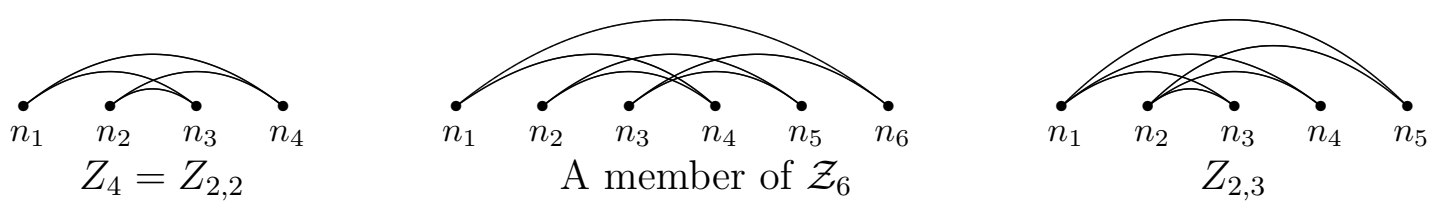

Figure 1: $Z_{4}=Z_{2,2}$, a member of $\mathcal{Z}_{6}$, and $Z_{2,3}$.

Any $n$-vertex $K_{s, t}$-free graph $G$ can be used to define a $Z_{s, t}$-free graph so ex $\left(n, K_{s, t}\right) \leqslant$ $\operatorname{ex}\left(n, Z_{s, t}\right)$. A non-trivial relation between $\operatorname{ex}\left(n, K_{s, t}\right)$ and $\operatorname{ex}\left(n, Z_{s, t}\right)$ can be viewed as a compactness result (see [12]) since one is forbidding a special type of $K_{s, t}$ rather than forbidding all $K_{s, t}$ 's. The same remark applies to zig-zag even cycles as well.

Our original motivation for investigating zig-zag bipartite graphs comes from a problem in additive number theory. A set $A \subset \mathbb{Z}$ is a $B_{2}$-set if $a_{1}+a_{2}=b_{1}+b_{2}$ with $a_{i}, b_{j} \in A$ implies $\left\{a_{1}, a_{2}\right\}=\left\{b_{1}, b_{2}\right\}$. $B_{2}$-sets, also called Sidon sets, were introduced in the early 1930 's and since then they have attracted the attention of many researchers. Let $F_{2}(n)$ be the maximum size of a $B_{2}$-set contained in $[n]$. Erdős and Turán [13] proved $F_{2}(n)<$ $n^{1 / 2}+O\left(n^{1 / 4}\right)$. In 1968 Lindström [22], refining the argument of Erdös and Turán, proved $F_{2}(n)<n^{1 / 2}+n^{1 / 4}+1$. Recently Cilleruelo [6] obtained $F_{2}(n)<n^{1 / 2}+n^{1 / 4}+1 / 2$ as a consequence of a more general result. Erdös conjectured $F_{2}(n)<n^{1 / 2}+O(1)$ and offered $\$ 500$ for a proof or disproof of this conjecture [10]. The error term of $n^{1 / 4}$ has not been improved since the original argument of Erdős and Turán.

The connection between $B_{2}$-sets and ordered Turán theory is given by the following construction. Let $A \subset[n]$ be a $B_{2}$-set and define the graph $G_{A}$ by $V\left(G_{A}\right)=[n]$ and

$$
E\left(G_{A}\right)=\{i j: i=j+a, a \in A\} .
$$

It is easily checked that $G_{A}$ is $Z_{4}$-free and so bounds on ex $\left(n, Z_{4}\right)$ translate to bounds on $F_{2}(n)$.

Our results are presented in the next section. Proofs are given in Sections 3, 4, and 5. In Section 6 we discuss the interaction between ordered Turán theory and $B_{2}$-sets. In the final section we make some concluding remarks.

\section{Results}

We begin our discussion with zig-zag even cycles. Before stating our result we recall some of the known bounds for $\operatorname{ex}\left(n, C_{2 k}\right)$. The first general upper bound on $\operatorname{ex}\left(n, C_{2 k}\right)$ is due to Bondy and Simonovits [3] who proved $\operatorname{ex}\left(n, C_{2 k}\right) \leqslant c_{k} n^{1+1 / k}$ where $c_{k}$ is a constant depending only on $k$. The best known upper bound on $\operatorname{ex}\left(n, C_{2 k}\right)$ for general $k$ is due to Pikhurko [25] who, using ideas of Verstraëte [26], showed

$$
\operatorname{ex}\left(n, C_{2 k}\right) \leqslant(k-1) n^{1+1 / k}+16(k-1) n .
$$

For $k \in\{2,3\}$ more precise results are known. By counting pairs of vertices in a common neighborhood, it is not hard to show $\operatorname{ex}\left(n, C_{4}\right) \leqslant \frac{1}{2} n^{3 / 2}+O(n)$ (see [23], Ch. 10, Problem 36). Graphs constructed independently by Erdős, Rényi [11] and Brown [5] show this 
upper bound is essentially best possible. Füredi, Naor, and Verstraëte [17] proved for sufficiently large $n, \operatorname{ex}\left(n, C_{6}\right) \leqslant 0.6272 n^{4 / 3}$. They also gave a construction which shows $\operatorname{ex}\left(n, C_{6}\right)>0.5338 n^{4 / 3}$ for infinitely many $n$. Lazebnik, Ustimenko, and Woldar [21], using a construction of Wenger [27], proved $\operatorname{ex}\left(n, C_{10}\right)>4 / 5^{6 / 5} n^{6 / 5}+o\left(n^{6 / 5}\right)$. To summarize, $\operatorname{ex}\left(n, C_{4}\right)$ is known asymptotically. For $k \in\{3,5\}$, the order of $\operatorname{ex}\left(n, C_{2 k}\right)$ is $n^{1+1 / k}$. For $k \notin\{2,3,5\}, \operatorname{ex}\left(n, C_{2 k}\right)$ is $O\left(n^{1+1 / k}\right)$ but there is no matching lower bound. Our first theorem gives an upper bound on $\operatorname{ex}\left(n, \mathcal{Z}_{2 k}\right)$.

Theorem 2. Let $k \geqslant 2$ be an integer. For any $n$,

$$
\operatorname{ex}\left(n, \mathcal{Z}_{2 k}\right) \leqslant \frac{k-3 / 2}{2^{1 / k}-1} n^{1+1 / k}+(2 k-3) n \log _{2} n .
$$

For $k=2$ the upper bound given by Theorem 2 will be improved by Theorem 3 . Using the bound $\operatorname{ex}\left(n, C_{2 k}\right) \leqslant \operatorname{ex}\left(n, \mathcal{Z}_{2 k}\right)$, Theorem 2 shows the order of magnitude of $\operatorname{ex}\left(n, \mathcal{Z}_{2 k}\right)$ is $n^{1+1 / k}$ for $k \in\{2,3,5\}$, but it is very unlikely that it is asymptotically optimal for any $k$. The constant $\frac{k-3 / 2}{2^{1 / k}-1}$ is asymptotic to $\frac{k^{2}}{\log 2}$ whereas the leading coefficient in the upper bound on $\operatorname{ex}\left(n, C_{2 k}\right)$ is linear in $k$.

Next we discuss zig-zag complete bipartite graphs. Given integers $n, m, t, s$ with $2 \leqslant$ $t \leqslant n$ and $2 \leqslant s \leqslant m$, let $z(n, m ; t, s)$ be the maximum number of 1 's in an $n$ by $m$ 0,1 -matrix that contains no $t$ by $s$ submatrix of all 1's. The problem of determining $z(n, m ; t, s)$ is known as the problem of Zarankiewicz. Improving an upper bound of Kövári, Sós, and Turán [20], Füredi [14] proved

$$
z(n, m ; t, s) \leqslant(t-s+1)^{1 / s} m n^{1-1 / s}+s m+s n^{2-2 / s}
$$

for all $n \geqslant t, m \geqslant s$, and $t \geqslant s \geqslant 1$. The connection between the Zarankiewicz problem and Turán theory is given by the inequality $\operatorname{ex}\left(n, K_{s, t}\right) \leqslant \frac{1}{2} z(n, n ; t, s)$ (see [2]) so that (1) implies

$$
\operatorname{ex}\left(n, K_{s, t}\right) \leqslant \frac{1}{2}(t-s+1)^{1 / s} n^{2-1 / s}+O\left(n^{2-2 / s}\right)
$$

for $t \geqslant s$. For lower bounds, a construction of Füredi [15] and (2) give $\operatorname{ex}\left(n, K_{2, t}\right)=$ $\frac{1}{2} \sqrt{t-1} n^{3 / 2}+o\left(n^{3 / 2}\right)$. A construction of Brown [5] and (2) give $\operatorname{ex}\left(n, K_{3,3}\right)=\frac{1}{2} n^{5 / 3}+$ $o\left(n^{5 / 3}\right)$. For other values of $s$ and $t$ the results are not as precise. When $t \geqslant(s-1) !+1$, graphs constructed by Kollár, Rónyai, and Szabó [19] (see also the paper of Alon, Rónyai, Szabó [1]) show $\operatorname{ex}\left(n, K_{s, t}\right) \geqslant c_{s, t} n^{2-1 / s}$. For other values of $s$ and $t$, there are no lower bounds that match (2) in order of magnitude.

Theorem 3 gives an upper bound on $\operatorname{ex}\left(n, Z_{s, t}\right)$ corresponding to (2).

Theorem 3. Let $t \geqslant s \geqslant 2$ be integers. For any $n$,

$$
\operatorname{ex}\left(n, Z_{s, t}\right) \leqslant \frac{(t-1)^{1 / s}}{2-1 / s} n^{2-1 / s}+\left((t-1)^{1 / s}+\frac{1}{2}(s-1)\right) n^{3 / 2-1 / 2 s}+(s-1) n .
$$


It is worth noting that for $s \leqslant t$,

$$
\lim _{s \rightarrow \infty} \lim _{t \rightarrow \infty} \frac{\frac{1}{2}(t-s+1)^{1 / s}}{(t-1)^{1 / s} /(2-1 / s)}=1 .
$$

For small values of $s$ and $t$ there is certainly a gap between the upper bounds on $\operatorname{ex}\left(n, K_{s, t}\right)$ and $\operatorname{ex}\left(n, Z_{s, t}\right)$. When $s=t=2$ the upper bound of Theorem 3 gives $\operatorname{ex}\left(n, Z_{2,2}\right) \leqslant \frac{2}{3} n^{3 / 2}+O\left(n^{5 / 4}\right)$ whereas $(2)$ gives $\operatorname{ex}\left(n, K_{2,2}\right) \leqslant \frac{1}{2} n^{3 / 2}+O(n)$.

We are able to give a construction using projective planes which shows

$$
\limsup _{n \rightarrow \infty}\left(\operatorname{ex}\left(n, Z_{4}\right)-\operatorname{ex}\left(n, C_{4}\right)\right)=\infty
$$

Unfortunately we were unable to determine whether or not $\operatorname{ex}\left(n, Z_{4}\right) \sim \operatorname{ex}\left(n, C_{4}\right)$ but we do have the following theorem.

Theorem 4. For any prime $p$,

$$
\operatorname{ex}\left(p^{2}+p+1, Z_{4}\right) \geqslant p^{2}+\operatorname{ex}\left(p^{2}+p+1, C_{4}\right) .
$$

The upper bound $\operatorname{ex}\left(n, Z_{4}\right) \leqslant \frac{2}{3} n^{3 / 2}+o\left(n^{3 / 2}\right)$ given by Theorem 3 can probably be improved. We believe the constructions are best possible.

Conjecture 5. The zig-zag Turán number ex $\left(n, Z_{4}\right)$ satisfies

$$
\operatorname{ex}\left(n, Z_{4}\right) \leqslant \frac{1}{2} n^{3 / 2}+o\left(n^{3 / 2}\right)
$$

The notion of compactness [12] has produced several interesting problems concerning Turán numbers for bipartite graphs. Similar questions can be asked for our ordered version of the problem.

Problem 6. Is it true that for any bipartite graph $H$ and any zig-zag $Z H$ we have

$$
\operatorname{ex}(n, Z H)=O(\operatorname{ex}(n, H)) ?
$$

A positive answer to Problem 6 is supported by Theorem 3.

Another interesting problem related to compactness is the following. Let $\mathcal{Z}_{2 k}^{\times}$be the sub-family of $\mathcal{Z}_{2 k}$ that consists of all $Z_{2 k}$ 's with a longest or shortest edge.

Problem 7. Is ex $\left(n, \mathcal{Z}_{2 k}^{\times}\right)=O\left(n^{1+1 / k}\right)$ for $k \geqslant 3$ ?

We will discuss Problem 7 in more detail in Section 7. For now we remark that it is not difficult to show $\operatorname{ex}\left(n, \mathcal{Z}_{2 k}^{\times}\right)>c n^{1+1 / k}$ for all $k \geqslant 3$ which may come as a surprise considering the difficulty in finding good lower bounds on $\operatorname{ex}\left(n, C_{2 k}\right)$ for $k \notin\{2,3,5\}$.

In the next three sections we will prove Theorems $2-4$. Throughout the paper all floor and ceiling symbols are omitted whenever they do not affect the asymptotics of the results. 


\section{Proof of Theorem 2}

Let $k \geqslant 2$ be an integer. Let $G$ be a $\mathcal{Z}_{2 k}$-free graph with $V(G)=[n]$. Given two subsets $A, B \subset V(G)$, write $A<B$ if all of the elements of $A$ are less than the smallest element of $B$. For disjoint subsets $A, B \subset V(G)$, let $G(A, B)$ be the subgraph of $G$ with $V(G(A, B))=A \cup B$ and

$$
E(G(A, B))=\{i j \in E(G): i \in A, j \in B\}
$$

For any pair of subsets $A, B \subset V(G)$ with $A<B$, the graph $G(A, B)$ is $C_{2 k}$-free since $G$ is $\mathcal{Z}_{2 k}$-free. Given integers $m_{1}$ and $m_{2}$, let $\operatorname{ex}\left(m_{1}, m_{2}, C_{2 k}\right)$ be the maximum number of edges in a $C_{2 k}$-free bipartite graph with $m_{1}$ vertices in one part and $m_{2}$ vertices in the other. Naor and Verstraëte [24] proved an upper bound on $\operatorname{ex}\left(m_{1}, m_{2}, C_{2 k}\right)$ that implies a $C_{2 k}$-free bipartite graph with $m$ vertices in each part has at most $(2 k-3)\left(m^{1+1 / k}+2 m\right)$ edges. Applying this bound to $G\left(A_{1}, B_{1}\right)$ where $A_{1}=\{1,2, \ldots, n / 2\}$ and $B_{1}=\{n / 2+$ $1, n / 2+2, \ldots, n\}$ gives

$$
e\left(G\left(A_{1}, B_{1}\right)\right) \leqslant(2 k-3)\left((n / 2)^{1+1 / k}+n\right) .
$$

We repeat the argument on the sets $A_{2,1}=\{1,2, \ldots, n / 4\}, B_{2,1}=\{n / 4+1, n / 4+$ $2, \ldots, n / 2\}$ and on the sets $A_{2,2}=\{n / 2+1, n / 2+2, \ldots, 3 n / 4\}, B_{2,2}=\{3 n / 4+1,3 n / 4+$ $2, \ldots, n\}$. Continuing in this fashion gives

$$
\begin{aligned}
e(G) & \leqslant \sum_{l=1}^{\log _{2} n} 2^{l-1} \operatorname{ex}\left(n 2^{-l}, n 2^{-l}, C_{2 k}\right) \\
& \leqslant \sum_{l=1}^{\log _{2} n} 2^{l-1}(2 k-3)\left(\left(n / 2^{l}\right)^{1+1 / k}+2 n / 2^{l}\right) \\
& \leqslant \frac{(k-3 / 2) n^{1+1 / k}}{2^{1 / k}} \sum_{l=0}^{\infty}\left(\frac{1}{2^{1 / k}}\right)^{l}+(2 k-3) n \log _{2} n \\
& =\frac{k-3 / 2}{2^{1 / k}-1} n^{1+1 / k}+(2 k-3) n \log _{2} n .
\end{aligned}
$$

\section{$4 \quad$ Proof of Theorem 3}

The following lemma was used by Füredi [14] to prove

$$
z(m, n ; t, s) \leqslant(t-s+1)^{1 / s} n m^{1-1 / s}+s n+s m^{2-2 / s}
$$

for all $m \geqslant t, n \geqslant s$ and $t \geqslant s \geqslant 2$. The proof of the lemma is an easy application of Jensen's Inequality. For $k \geqslant 1$ and $x \geqslant k-1$ define $\left(\begin{array}{l}x \\ k\end{array}\right)=\frac{1}{k !} x(x-1) \cdots(x-k+1)$. If $k-1>x \geqslant 0$ define $\left(\begin{array}{l}x \\ k\end{array}\right)=0$. For fixed $k$ each of these functions is convex. 
Lemma 8 (Füredi, [14]). If $n, k \geqslant 1$ are integers and $c, y, x_{1}, \ldots, x_{k}$ are non-negative real numbers and $\sum_{i=1}^{n}\left(\begin{array}{c}x_{i} \\ k\end{array}\right) \leqslant c\left(\begin{array}{l}y \\ k\end{array}\right)$ then

$$
\sum_{i=1}^{n} x_{i} \leqslant y c^{1 / k} n^{1-1 / k}+(k-1) n .
$$

Proof. Let $s=\sum_{i=1}^{n} x_{i}$. If $s \leqslant n(k-1)$ then the inequality holds so assume $\frac{s}{n}-k+1>$ 0 . Apply Jensen's Inequality to get $\sum_{i=1}^{n}\left(\begin{array}{c}x_{i} \\ k\end{array}\right) \geqslant n\left(\begin{array}{c}s / n \\ k\end{array}\right)$ which implies $c\left(\begin{array}{l}y \\ k\end{array}\right) \geqslant n\left(\begin{array}{c}s / n \\ k\end{array}\right)$. Rearranging this inequality gives

$$
\frac{y(y-1)(y-2) \cdots(y-k+1)}{(s / n)(s / n-1) \cdots(s / n-k+1)} \geqslant \frac{n}{c} .
$$

The left hand side can be bounded above by $\left(\frac{y}{s / n-k+1}\right)^{k}$ to get $\left(\frac{y}{s / n-k+1}\right)^{k} \geqslant \frac{n}{c}$. Solving this inequality for $s$ gives (3).

Define the back neighborhood of a vertex $i \in V(G)$ to be the set

$$
\Gamma^{-}(i)=\{j<i: j i \in E(G)\} .
$$

Let $G$ be a $Z_{s, t}$-free graph with $V(G)=\{1,2, \ldots, n\}$. Define an $n$ by $n$ bipartite graph $H$ with parts $L=\left\{b_{1}, b_{2}, \ldots, b_{n}\right\}, P=\{1,2, \ldots, n\}$, and edge set

$$
E(H)=\left\{\left\{i, b_{j}\right\}: i \in \Gamma^{-}(j)\right\} .
$$

$H$ is the incidence graph of the back neighborhoods $\left\{\Gamma^{-}(i)\right\}_{i=1}^{n}$ of $G$.

It is easy to check that $e(H)=e(G)$ and $H$ has no complete bipartite subgraph with $t$ vertices in $L$ and $s$ vertices in $P$. Let $k=n^{1 / 2-1 / 2 s}$. For $j=1,2, \ldots, k$ let

$$
P_{j}=\left\{1+(j-1) \frac{n}{k}, 2+(j-1) \frac{n}{k}, \ldots, \frac{j n}{k}\right\} .
$$

Any back neighborhood $\Gamma^{-}(i)$ is a subset of $\{1,2, \ldots, i-1\}$ and so the neighbors of $b_{i}$ in $H$ are contained in the set $\{1,2, \ldots, i-1\}$. If $i<(j-1) \frac{n}{k}+1$ then $d_{P_{j}}\left(b_{i}\right)=0$ hence

$$
e\left(L, P_{j}\right)=\sum_{i=1}^{n} d_{P_{j}}\left(b_{i}\right)=\sum_{i=1+(j-1) \frac{n}{k}}^{n} d_{P_{j}}\left(b_{i}\right) .
$$

Recall $\left(\begin{array}{l}x \\ s\end{array}\right)=0$ if $0 \leqslant x<s$ and so

$$
\sum_{i=1}^{n}\left(\begin{array}{c}
d_{P_{j}}\left(b_{i}\right) \\
s
\end{array}\right)=\sum_{i=(j-1) \frac{n}{k}+1}^{n}\left(\begin{array}{c}
d_{P_{j}}\left(b_{i}\right) \\
s
\end{array}\right) .
$$

Each subset of size $s$ in $P_{j}$ can be counted at most $t-1$ times in the sum $\sum_{i=1}^{n}\left({ }_{P_{j}}\left(b_{i}\right)\right)$ therefore

$$
(t-1)\left(\begin{array}{c}
n / k \\
s
\end{array}\right) \geqslant \sum_{i=1}^{n}\left(\begin{array}{c}
d_{P_{j}}\left(b_{i}\right) \\
s
\end{array}\right)=\sum_{i=1+(j-1) \frac{n}{k}}^{n}\left(\begin{array}{c}
d_{P_{j}}\left(b_{i}\right) \\
s
\end{array}\right) .
$$


By Lemma 8,

$$
\sum_{i=1+(j-1) \frac{n}{k}}^{n} d_{P_{j}}\left(b_{i}\right) \leqslant \frac{n}{k}(t-1)^{1 / s}\left(n\left(1-\frac{j-1}{k}\right)\right)^{1-1 / s}+(s-1)\left(n\left(1-\frac{j-1}{k}\right)\right) .
$$

Using (4) and (5) we obtain

$$
\begin{aligned}
e(H) & =\sum_{j=1}^{k} e\left(L, P_{j}\right) \\
& \leqslant \frac{(t-1)^{1 / s} n^{2-1 / s}}{k} \sum_{j=1}^{k}(1-(j-1) / k)^{1-1 / s}+(s-1) n \sum_{j=1}^{k}(1-(j-1) / k) \\
& \leqslant \frac{(t-1)^{1 / s} n^{2-1 / s}}{k}\left(1+\int_{0}^{k}(1-x / k)^{1-1 / s} d x\right)+(s-1) n\left(1+\int_{0}^{k}(1-x / k) d x\right) \\
& =\frac{(t-1)^{1 / s}}{2-1 / s} n^{2-1 / s}+\frac{(t-1)^{1 / s} n^{2-1 / s}}{k}+\frac{(s-1) n k}{2}+(s-1) n \\
& =\frac{(t-1)^{1 / s}}{2-1 / s} n^{2-1 / s}+\left((t-1)^{1 / s}+\frac{1}{2}(s-1)\right) n^{3 / 2-1 / 2 s}+(s-1) n .
\end{aligned}
$$

Since $e(G)=e(H)$, this completes the proof.

\section{A lower bound}

Graphs constructed by Erdős, Rényi [11] and Brown [5] show ex $\left(q^{2}+q+1, C_{4}\right) \geqslant \frac{1}{2} q(q+1)^{2}$ where $q$ is any odd prime power. Since $\operatorname{ex}\left(n, Z_{4}\right) \geqslant \operatorname{ex}\left(n, C_{4}\right)$, this implies

$$
\operatorname{ex}\left(n, Z_{4}\right) \geqslant \frac{1}{2} n^{3 / 2}-o\left(n^{3 / 2}\right)
$$

The construction we present improves this lower bound in the error term. Füredi [16] proved ex $\left(q^{2}+q+1, C_{4}\right) \leqslant \frac{1}{2} q(q+1)^{2}=\frac{1}{2} q^{3}+q^{2}+\frac{1}{2} q$ for $q \geqslant 15$. Using the constructions of Erdös, Rényi, and Brown we have the exact result $\operatorname{ex}\left(q^{2}+q+1, C_{4}\right)=\frac{1}{2} q(q+1)^{2}$ for prime power $q$. For each prime $p$, we construct a $Z_{4}$-free graph with $p^{2}+p+2$ vertices, maximum degree $p+1$, and $\frac{1}{2} p^{3}+2 p^{2}+\frac{3}{2} p+1$ edges. It follows that there exists a $Z_{4}$-free graph on $p^{2}+p+1$ vertices with at least $\frac{1}{2} p^{3}+2 p^{2}+\frac{1}{2} p$ edges and so for prime $p \geqslant 15$,

$$
\operatorname{ex}\left(p^{2}+p+1, C_{4}\right)+p^{2} \leqslant \operatorname{ex}\left(p^{2}+p+1, Z_{4}\right) .
$$

Before giving the construction we point out a connection between $Z_{4}$-free graphs on $n$-vertices and collections subsets of $[n]$. Let $G$ be a $Z_{4}$-free graph on $[n]$. The back neighborhoods $\left\{\Gamma^{-}(i)\right\}_{i=2}^{n}$ form a collection of subsets of $[n]$ that satisfy

1. $\Gamma^{-}(i) \subseteq[i-1]$ for $2 \leqslant i \leqslant n$. 
2. For any $i \neq j,\left|\Gamma^{-}(i) \cap \Gamma^{-}(j)\right| \leqslant 1$.

Conversely any collection of sets $\left\{A_{i}\right\}_{i=1}^{n-1}$ that satisfy $A_{i} \subset[i]$ and $\left|A_{i} \cap A_{j}\right| \leqslant 1$ for $i \neq j$ can be used to define a $Z_{4}$-free graph $G$ by setting $\Gamma^{-}(i+1)=A_{i}$. We will construct a family of sets $A_{1}, \ldots, A_{p^{2}+p+1}$ that satisfies these conditions by labeling the points of a projective plane using the numbers $1,2, \ldots, p^{2}+p+1$ and by labeling the lines of the plane using the numbers $1,2, \ldots, p^{2}+p+1$. Suppose $l$ is a line that is assigned label $i$ and we denote this by $l_{i}$. Our goal is to make the sum

$$
\sum_{i=1}^{p^{2}+p+1}\left|l_{i} \cap[i]\right|
$$

as large as possible for if $G$ is defined by setting $\Gamma^{-}(i+1)=l_{i} \cap[i]$ for $1 \leqslant i \leqslant p^{2}+p+1$, then

$$
e(G)=\sum_{i=2}^{p^{2}+p+2}\left|\Gamma^{-}(i)\right|=\sum_{i=1}^{p^{2}+p+1}\left|l_{i} \cap[i]\right| .
$$

Now we proceed with the construction. Fix a prime $p$. The number $p^{2}+p+1$ and the numbers in the array

$$
\begin{array}{lllll}
p^{2}+p & p^{2}+p-1 & p^{2}+p-2 & \ldots & p^{2}+1 \\
p^{2} & p^{2}-1 & p^{2}-2 & \ldots & p^{2}-p+1 \\
\vdots & \vdots & \vdots & \ldots & \vdots \\
p & p-1 & p-2 & \ldots & 1
\end{array}
$$

are the points of the projective plane. To define the lines of the plane it is convenient to let $a=p^{2}+p+1$ and let $a_{i-1, j}$ be the $(i, j)$-entry in the array above. The lines of the plane are $l(r)=\left\{a, a_{r, 1}, a_{r, 2}, \ldots, a_{r, p}\right\}$ where $0 \leqslant r \leqslant p$, and $l(r, c)=\left\{a_{0, r}, a_{1, r+c}, a_{2, r+2 c}, \ldots, a_{p, r+p c}\right\}$ where $1 \leqslant r, c \leqslant p$. The second subscript $r+j c$ is reduced modulo $p$ so that its value is in $\{1,2, \ldots, p\}$. These names are used only to define the lines and at this point we are ready to assign the labels $1,2, \ldots, p^{2}+p+1$ to the lines. Each label will be used exactly once and there are $p^{2}+p+1$ lines so to label the lines we just drop the name after the label has been assigned. Give $l(0)$ label $p^{2}+p+1$ and for $1 \leqslant r \leqslant p$, give $l(r)$ label $p^{2}-p(r-1)$.

$$
l(0) \rightarrow l_{p^{2}+p+1} \quad l(r) \rightarrow l_{p^{2}-p(r-1)} \text { for } 1 \leqslant r \leqslant p .
$$

To determine the label assigned to $l(r, c)$, we look at which point $l(r, c)$ contains from the $(c+1)$-st row of the array. This point, or more precisely its label, will be the label assigned to $l(r, c)$ unless this element is a multiple of $p$. Specifically for $1 \leqslant r, c \leqslant p$,

$$
l(r, c) \rightarrow \begin{cases}l_{a_{c, r+c^{2}}} & \text { if } a_{c, r+c^{2}}<p^{2}-(c-1) p, \\ l_{p^{2}+p-(c-1)} & \text { if } a_{c, r+c^{2}}=p^{2}-(c-1) p .\end{cases}
$$

Before going further an example is needed. For $p=3$ form the array 


$\begin{array}{ccc}12 & 11 & 10 \\ 9 & 8 & 7 \\ 6 & 5 & 4 \\ 3 & 2 & 1\end{array}$

In this case $a=13, a_{0,1}=12, a_{0,2}=11, a_{0,3}=10, a_{1,1}=9, \ldots, a_{3,3}=1$. Below we show the lines before the label assignments are given and the new labels. For lines of the form $l(r, c)$ we have underlined the point of the line used to determine its label.

$$
\begin{array}{lll}
l(0)=\{13,12,11,10\} \rightarrow l_{13} & l(1)=\{13,9,8,7\} \rightarrow l_{9} & l(2)=\{13,6,5,4\} \rightarrow l_{6} \\
l(3)=\{13,3,2,1\} \rightarrow l_{3} & & \\
l(1,1)=\{12, \underline{8}, 4,3\} \rightarrow l_{8} & l(1,2)=\{11,7, \underline{5}, 3\} \rightarrow l_{5} & l(1,3)=\{10,9,6, \underline{3}\} \rightarrow l_{10} \\
l(2,1)=\{12, \underline{\underline{7}}, 6,2\} \rightarrow l_{7} & l(2,2)=\{11,9, \underline{4}, 2\} \rightarrow l_{4} & l(2,3)=\{10,8,5, \underline{2}\} \rightarrow l_{2} \\
l(3,1)=\{12, \underline{9}, 5,1\} \rightarrow l_{12} & l(3,2)=\{11,8, \underline{6}, 1\} \rightarrow l_{11} & l(3,3)=\{10,7,4, \underline{1}\} \rightarrow l_{1}
\end{array}
$$

To compute $\sum_{i=1}^{p^{2}+p+1}\left|l_{i} \cap[i]\right|$, we divide it into three smaller sums. It is easy to check

$$
\sum_{i=1}^{p}\left|l_{i p} \cap[i p]\right|=p \cdot p
$$

and

$$
\sum_{i=p^{2}+1}^{p^{2}+p+1}\left|l_{i} \cap[i]\right|=(p+1)(p+1) .
$$

For fixed $j$ with $0 \leqslant j \leqslant p-1$,

$$
\sum_{i=j p+1}^{(j+1) p-1}\left|l_{i} \cap[i]\right|=(p-1)(j+1) .
$$

Putting (6), (7), and (8) together gives

$$
\sum_{i=1}^{p^{2}+p+1}\left|l_{i} \cap[i]\right|=p^{2}+(p+1)^{2}+(p-1) \sum_{j=1}^{p} j=\frac{1}{2} p^{3}+2 p^{2}+\frac{3}{2} p+1 .
$$

We summarize this construction as a result on labeling points and lines of a projective plane. Define a labeling of a projective plane $(\mathcal{P}, \mathcal{L})$ of order $q$ to be a pair of bijections $L_{\mathcal{P}}: \mathcal{P} \rightarrow\left\{1,2, \ldots, q^{2}+q+1\right\}$ and $L_{\mathcal{L}}: \mathcal{L} \rightarrow\left\{l_{1}, l_{2}, \ldots, l_{q^{2}+q+1}\right\}$.

Proposition 9. Let $(\mathcal{P}, \mathcal{L})$ be a projective plane of order $p$ where $p$ is prime. There is a labeling of the points $L_{\mathcal{P}}: \mathcal{P} \rightarrow\left\{1,2, \ldots, p^{2}+p+1\right\}$ and the lines $L_{\mathcal{L}}: \mathcal{L} \rightarrow$ $\left\{l_{1}, l_{2}, \ldots, l_{p^{2}+p+1}\right\}$ such that

$$
\sum_{i=1}^{p^{2}+p+1}\left|l_{i} \cap[i]\right|=\frac{1}{2} p^{3}+2 p^{2}+\frac{3}{2} p+1 .
$$


An easier way to obtain a labeling of a projective plane of order $q$ is to label the points and lines randomly. The sum $X=\sum_{i=1}^{q^{2}+q+1}\left|l_{i} \cap[i]\right|$ is a random variable whose expectation and variance can be computed exactly as $\mathbb{E} X=\frac{1}{2} q^{3}+q^{2}+\frac{3}{2} q+1$ and $\operatorname{Var} X=\frac{n}{12} \sqrt{n-3 / 4}-\frac{n}{24}+\frac{1}{12} \sqrt{n-3 / 4}-\frac{1}{24}$ where $n=q^{2}+q+1$. Furthermore $X$ has a nice symmetry property that allows one to prove that there are outcomes where $X \geqslant \mathbb{E} X+\frac{1}{2} \sqrt{\operatorname{Var} X}$. This method produces a labeling with $X \geqslant \frac{1}{2} q^{3}+q^{2}+O\left(q^{1.5}\right)$. When $q$ is prime this matches our construction in the leading term but is not as good in second term. On the other hand, we do not know of any other method to label projective planes whose order is not a prime.

One may suspect that with some clever labeling we can find a sequence of projective planes of order $q_{1}<q_{2}<\ldots$ such that

$$
\sum_{i=1}^{q_{k}^{2}+q_{k}+1}\left|l_{i} \cap[i]\right|>\left(\frac{1}{2}+\epsilon\right) q_{k}^{3}
$$

for a fixed $\epsilon>0$. The next result shows that this cannot be done.

Proposition 10. Let $(\mathcal{P}, \mathcal{L})$ be a projective plane of order $q$. If $L_{\mathcal{P}}, L_{\mathcal{L}}$ is a labeling of $(\mathcal{P}, \mathcal{L})$ then

$$
\sum_{i=1}^{q^{2}+q+1}\left|l_{i} \cap[i]\right|=\frac{1}{2} q^{3}+o\left(q^{3}\right) .
$$

To prove Proposition 10 we need the following lemma (see [18]).

Lemma 11. Let $G$ be a d-regular, $n$-vertex bipartite graph with parts $X, Y$ and set $\lambda=$ $\max _{i \neq 1, n}\left|\lambda_{i}\right|$ where $\lambda_{1} \geqslant \ldots \geqslant \lambda_{n}$ are the eigenvalues of the adjacency matrix of $G$. For any $S \subset X, T \subset Y$,

$$
\left|e(S, T)-\frac{2 d|S||T|}{n}\right| \leqslant \frac{\lambda}{2}(|S|+|T|)
$$

Proof of Proposition 10. Let $(\mathcal{P}, \mathcal{L})$ be a projective plane of order $q$ and let $L_{\mathcal{P}}, L_{\mathcal{L}}$ be a labeling of $(\mathcal{P}, \mathcal{L})$. Let $t=q^{1 / 4}$ and for $1 \leqslant i \leqslant t$, let $S_{i}=\left\{1,2, \ldots, \frac{i q^{2}}{t}\right\}$ and

$$
T_{i}=\left\{l_{1+(i-1) \frac{q^{2}}{t}}, l_{2+(i-1) \frac{q^{2}}{t}}, \ldots, l_{\frac{q^{2}}{t}+(i-1) \frac{q^{2}}{t}}\right\} .
$$

Let $A$ be the adjacency matrix of the incidence graph of $(\mathcal{P}, \mathcal{L})$. The eigenvalues of $A$ are $q+1,-(q+1)$, each with multiplicity 1 , and all other eigenvalues are $\pm \sqrt{q}$. This can be seen by considering the matrix $A^{2}$ which has the rather simple form $A^{2}=\left(\begin{array}{cc}B & 0 \\ 0 & B\end{array}\right)$ where $B=I+q J$ and $J$ is the all 1's matrix. Using Lemma 11,

$$
\sum_{i=1}^{q^{2}+q+1}\left|l_{i} \cap[i]\right| \leqslant(q+1)^{2}+\sum_{i=1}^{q^{2}}\left|l_{i} \cap[i]\right| \leqslant(q+1)^{2}+\sum_{i=1}^{t} e\left(S_{i}, T_{i}\right)
$$




$$
\begin{aligned}
& \leqslant(q+1)^{2}+\sum_{i=1}^{t}\left(\frac{2(q+1) q^{4} i}{2\left(q^{2}+q+1\right) t^{2}}+\frac{\sqrt{q}}{2}\left(\frac{i q^{2}}{t}+\frac{q^{2}}{t}\right)\right) \\
& \leqslant(q+1)^{2}+\frac{q^{4}(t+1)}{2(q-1) t}+\frac{q^{5 / 2}}{2}+\frac{q^{5 / 2}(t+1)}{4} \\
& =\frac{1}{2} q^{3}+o\left(q^{3}\right) .
\end{aligned}
$$

A similar argument gives the lower bound $\sum_{i=1}^{q^{2}+q+1}\left|l_{i} \cap[i]\right| \geqslant \frac{1}{2} q^{3}+o\left(q^{3}\right)$.

Proof of Theorem 4. By Proposition 9, there exists a $Z_{4}$-free graph $G_{p}$ with $p^{2}+p+2$ vertices and $\frac{1}{2} p^{3}+2 p^{2}+\frac{3}{2} p+1$ edges for prime $p$. Furthermore this graph has maximum degree $p+1$. Let $G_{p}^{\prime}$ be a subgraph of $G_{p}$ with $p^{2}+p+1$ vertices and at least $\frac{1}{2} p^{3}+2 p^{2}+\frac{1}{2} p$ edges. Then

$$
\begin{aligned}
\operatorname{ex}\left(p^{2}+p+1, Z_{4}\right) & \geqslant e\left(G_{p}^{\prime}\right) \geqslant \frac{1}{2} p^{3}+2 p^{2}+\frac{1}{2} p \\
& =\operatorname{ex}\left(p^{2}+p+1, C_{4}\right)+p^{2}
\end{aligned}
$$

\section{$6 \quad B_{2}$-sets and $Z_{4}$-free graphs}

In this section we show how $B_{2}$-sets can be used to construct $Z_{4}$-free graphs. Let $A \subset[n]$ be a $B_{2}$-set. Define the graph $G_{A}$ by $V\left(G_{A}\right)=[n]$ and

$$
E\left(G_{A}\right)=\{i j: i=j+a, a \in A\} .
$$

Perhaps the most interesting feature of this construction is that, in general, $G_{A}$ will contain many $C_{4}$ 's but will still be $Z_{4}$-free. For each vertex $i$ and pair $\{a, b\} \subset A$ with $i+a+b \leqslant n$, the vertices $\{i, i+a, i+b, i+a+b\}$ form a $C_{4}$ in $G$.

Lemma 12. If $A \subset[n]$ is a $B_{2}$-set, then $G_{A}$ is a $Z_{4}$-free graph with $\sum_{i=1}^{n-1}|A \cap[i]|$ edges.

Proof. Suppose $n_{1}<n_{2}<n_{3}<n_{4}$ are the vertices of a $Z_{4}$ in $G$. There exists $a, b, c, d \in A$ such that $n_{3}=a+n_{1}, n_{4}=b+n_{1}, n_{3}=c+n_{2}$, and $n_{4}=d+n_{2}$. This implies $a-c+d-b=0$ so that $\{a, d\}=\{b, c\}$. If $a=b$ then $n_{3}=n_{4}$ and if $a=c$ then $n_{1}=n_{2}$. In either case we have a contradiction.

The number of edges of $G_{A}$ is $\sum_{i=1}^{n}\left|\Gamma^{-}(i)\right|$ and $\left|\Gamma^{-}(i)\right|=|A \cap[i-1]|$.

Observe that for any such $G_{A}$,

$$
\operatorname{ex}\left(n, Z_{4}\right) \geqslant e\left(G_{A}\right)
$$

If we can accurately estimate $\sum_{i=1}^{n-1}|A \cap[i]|$ then upper bounds on $\operatorname{ex}\left(n, Z_{4}\right)$ can imply upper bounds on $|A|$. We can use the following result of Cilleruelo to show $e\left(G_{A}\right)=$ $\frac{1}{2} n^{3 / 2}-o\left(n^{3 / 2}\right)$ provided $A$ is chosen appropriately. 
Theorem 13 (Cilleruelo, [7]). If $A \subset[n]$ is a $B_{2}$-set with $n^{1 / 2}-L$ elements then any interval of length cn contains $c|A|+e_{I}$ elements of $A$ where

$$
\left|e_{I}\right| \leqslant 52 n^{1 / 4}\left(1+c^{1 / 2} n^{1 / 8}\right)\left(1+L_{+}^{1 / 2} n^{-1 / 8}\right)
$$

and $L_{+}=\max \{0, L\}$.

Theorem 14. For each $B_{2}$-set $A$ with $|A|=n^{1 / 2}$ there exists an n-vertex $Z_{4}$-free graph $G_{A}$ with

$$
e\left(G_{A}\right) \geqslant \frac{1}{2} n^{3 / 2}-O\left(n^{5 / 4}\right) .
$$

Furthermore $G_{A}$ has at least $\frac{n^{2}}{18}-O\left(n^{15 / 8}\right)$ 4-cycles.

Proof. Suppose $A \subset[n]$ is a $B_{2}$-set with $|A|=n^{1 / 2}$. Let $k=n^{1 / 4}$ and for $1 \leqslant j \leqslant k$, let

$$
P_{j}=\left\{1+\frac{(j-1) n}{k}, 2+\frac{(j-1) n}{k}, \ldots, \frac{j n}{k}\right\} .
$$

Using Theorem 13,

$$
\sum_{i=1}^{n}|A \cap[i]| \geqslant \sum_{j=2}^{k} \sum_{i \in P_{j}}|A \cap[i]| \geqslant \sum_{j=2}^{k} \frac{n}{k}\left|A \cap\left[\frac{(j-1) n}{k}\right]\right|=\frac{n}{k} \sum_{j=2}^{k}\left(\frac{j-1}{k}|A|+e_{I_{j}}\right)
$$

where $e_{I_{j}}$ satisfies the inequality

$$
e_{I_{j}} \geqslant-52 n^{1 / 4}\left(1+\sqrt{\frac{j-1}{k}} n^{1 / 8}\right) .
$$

Now $\frac{n}{k} \sum_{j=2}^{k} \frac{j-1}{k}|A|=\frac{1}{2} n^{3 / 2}-\frac{n^{3 / 2}}{2 k}$ and so it remains to find a lower bound on the sum $-\frac{52 n^{5 / 4}}{k} \sum_{j=1}^{k-1}\left(1+\sqrt{j / k} n^{1 / 8}\right)$. Estimating the sum with an integral gives

$$
-\frac{52 n^{5 / 4}}{k} \sum_{j=1}^{k-1}\left(1+\sqrt{j / k} n^{1 / 8}\right) \geqslant-\frac{52 n^{5 / 4}}{k}\left(k+\frac{2 n^{1 / 8} k}{3}\right)=-52 n^{5 / 4}-\frac{104 n^{11 / 8}}{3} .
$$

Thus

$$
\sum_{i=1}^{n}|A \cap[i]| \geqslant \frac{1}{2} n^{3 / 2}-\frac{n^{3 / 2}}{2 k}-52 n^{5 / 4}-\frac{104 n^{11 / 8}}{3} \geqslant \frac{1}{2} n^{3 / 2}-O\left(n^{5 / 4}\right) .
$$

To prove the statement concerning 4-cycles, observe Theorem 13 implies

$$
|A \cap[1, n / 3]| \geqslant \frac{1}{3} n^{1 / 2}-104 n^{3 / 8} .
$$

For any vertex $i \in[1, n / 3]$ and pair $\{a, b\} \subset A \cap[1, n / 3]$, the vertices $\{i, i+a, i+b, i+a+b\}$ form a 4 -cycle. If $\alpha=\frac{1}{3} n^{1 / 3}-104 n^{3 / 8}$, then there are $\frac{n}{3}\left(\begin{array}{c}\alpha \\ 2\end{array}\right)=\frac{n^{2}}{18}-O\left(n^{15 / 8}\right)$ such 4 -cycles.

For $q$ a prime power, there exists $B_{2}$-sets $A \subset\left[q^{2}\right]$ with $|A|=q$ (see [4]). Applying Theorem 14 to such a $B_{2}$-set gives a $Z_{4}$-free graph with $\frac{1}{2} n^{3 / 2}+o\left(n^{3 / 2}\right)$ edges where $n=q^{2}$. 


\section{Concluding Remarks}

- One might hope that the idea of using $B_{2}$-sets to construct $Z_{4}$-free graphs extends to using $B_{k}$-sets to construct $\mathcal{Z}_{2 k}$-free graphs. A set $A \subset \mathbb{Z}$ is a $B_{k}$-set if whenever

$$
a_{1}+a_{2}+\cdots+a_{k}=b_{1}+b_{2}+\cdots+b_{k} \text { with } a_{i}, b_{j} \in A,
$$

the elements $a_{1}, a_{2}, \ldots, a_{k}$ are a permutation of $b_{1}, b_{2}, \ldots, b_{k}$. Unfortunately this does not work in general. If $A \subset[n]$ is a $B_{k}$-set with $k \geqslant 3$, then $G_{A}$, defined the same way as in Section 6 , may not be $\mathcal{Z}_{2 k}$-free. For example, if $a>b>c$ are elements of $A$ with $a-c<b$ then the $Z_{6}$ shown in Figure 2 can appear in $G_{A}$.

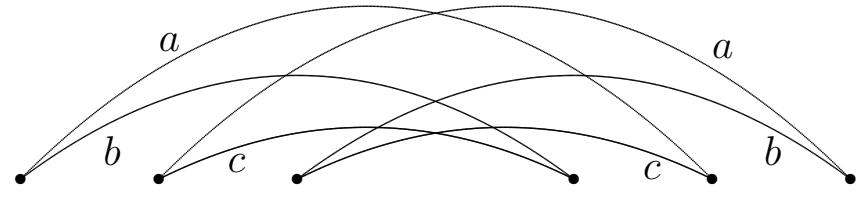

Figure 2: $Z_{6}$ in $G_{A}$

For each such triple, there are $n-(a+b-c)$ choices for the first vertex so that deleting one edge from each such $Z_{6}$ will remove too many edges. The case for longer even cycles is more complicated.

If we forbid a subfamily of $Z_{2 k}$ 's then $B_{k}$-sets can be used to give good constructions. Let $A \subset[n]$ be a $B_{k}$-set with $k \geqslant 3$ and consider a $Z_{2 k}$ in $G_{A}$. Suppose this $Z_{2 k}$ is $x_{1} y_{1} x_{2} y_{2} \ldots x_{k} y_{k} x_{1}$ where $x_{i}<y_{j}$ for all $i, j$. Since $A$ is a $B_{k}$-set,

$$
\left\{y_{1}-x_{1}, y_{2}-x_{2}, \ldots, y_{k}-x_{k}\right\}=\left\{y_{1}-x_{2}, y_{2}-x_{3}, \ldots, y_{k}-x_{1}\right\}
$$

and so we cannot have an edge that is longer or shorter than all of the other edges. Recall $\mathcal{Z}_{2 k}^{\times}$is the family of $Z_{2 k}$ 's with a longest or shortest edge. Using $B_{k}$-sets constructed in [4], we obtain the lower bound

$$
\operatorname{ex}\left(n, \mathcal{Z}_{2 k}^{\times}\right) \geqslant c n^{1+1 / k}
$$

where $c>0$ is a constant independent of $k$. The difficulty now lies in proving good upper bounds which is the content of Problem 7 .

- The argument used to prove Theorem 2 can be generalized. If $F$ is a bipartite graph with a unique bipartition and an automorphism interchanging the parts then we can obtain an upper bound on $\operatorname{ex}(n, \mathcal{Z F})$ in terms of ex $(n, F)$ where $\mathcal{Z F}$ is the family of zig-zag versions of $F$.

More precisely, if $\operatorname{ex}(n, F) \leqslant c n^{\delta}$ for some constant $c>0$ and real $\delta \in(1,2)$, then we can show $\operatorname{ex}(n, \mathcal{Z F}) \leqslant \frac{2^{\delta-1} c}{2^{\delta-1}-1} n^{\delta}$. For instance, using the bound $\operatorname{ex}\left(n, C_{6}\right)<0.6272 n^{4 / 3}$ for large $n$ [17], we get $\operatorname{ex}\left(n, \mathcal{Z}_{6}\right)<\frac{2^{1 / 3}}{2^{1 / 3}-1} \cdot 0.6272 n^{4 / 3}<3.0403 n^{4 / 3}$ for large $n$ which is an improvement of Theorem 2. 


\section{Acknowledgements}

The author would like to thank Jacques Verstraëte for his many helpful suggestions and André Kündgen for comments that improved the presentation of the paper.

\section{References}

[1] N. Alon, L. Rónyai, T. Szabó, Norm-graphs: variations and applications, J. Combinatorial Theory B, 76 (2) (1999), p. 280-290.

[2] B. Bollobás, Extremal Graph Theory, Academic Press, London - New York, 1978.

[3] J. A. Bondy, M. Simonovits, Cycles of even length in graphs, J. Combinatorial Theory B, 16 (1974), p. 97-105.

[4] R. C. Bose, S. Chowla, Theorems in the additive theory of numbers, Comment. Math. Helv. 37 (1962/1963), p. 141-147.

[5] W. G. Brown, On graphs that do not contain a Thomsen graph, Canad. Math. Bull. 9 (1966), p. 281-289.

[6] J. Cilleruelo, Sidon sets in $\mathbb{N}^{d}$, J. of Combinatorial Theory Series A, 117 (2010), p. 857-871.

[7] J. Cilleruelo, Gaps in dense Sidon sets, Integers 11 (2001) A11.

[8] J. Czipszer, P. Erdős, A. Hajnal, Some extremal problems on infinite graphs, Publications of the Math. Inst. of the Hungarian Academy of Sci. Ser. A 7 (1962), p. 441456.

[9] A. Dudek, V. Rödl, On the Turán properties of infinite graphs, Electronic J. of Combinatorics 15 (2008) \#R47.

[10] P. Erdős, A survey of problems in combinatorial number theory, Annals of Discrete Mathematics 6 (1980), p. 89-115.

[11] P. Erdős, A. Rényi, On a problem in the theory of graphs, Publ. Math. Inst. Hungar. Acad. Sci. 7A (1962), p. 623-641.

[12] P. Erdős, M. Simonovits, Compactness results in extremal graph theory, Combinatorica 2 (3) (1982), p. 275-288.

[13] P. Erdős, P. Turán, On a problem of Sidon in additive number theory, and on some related problems, J. London Math. Soc. 16 (1941), p. 212-215.

[14] Z. Füredi, An upper bound on Zarankiewicz' Problem, Combinatorics, Probability and Computing 5 (1996), p. 29-33.

[15] Z. Füredi, New asymptotics for bipartite Turán numbers, J. of Combinatorial Theory Series A, 75 (1) (1996), p. 141-144.

[16] Z. Füredi, On the number of edges of quadrilateral-free graphs, J. Combinatorial Theory B, 681 (1974), p. 1-6. 
[17] Z. Füredi, A. Naor, J. Verstraëte, On the Turán number for the hexagon, Adv. Math. 203 (2006), no. 2, p. 476-496.

[18] P. Keevash, B. Sudakov, J. Verstraëte, On a conjecture of Erdős and Simonovits: Even Cycles, arXiv:1107.4715.

[19] J. Kollár, L. Rónyai, T. Szabó, Norm-graphs and bipartite Turán numbers, Combinatorica, 16 (3) (1996), p. 399-406.

[20] T. Kövári, V. T. Sós, P. Turán, On a problem of Zarankiewicz, Colloq. Math. 3 (1954), p. 50-57.

[21] F. Lazebnik, V. A. Ustimenko, A. J. Woldar, Properties of certain families of $2 k$ cycle-free graphs, J. of Combinatorial Theory Series B, 602 (1994), p. 293-298.

[22] B. Lindström, An inequality for $B_{2}$-sequences, J. Combinatorial Theory 6 (1969), p. 211-212.

[23] L. Lovász, Combinatorial Problems and Exercises, AMS Chelsea Publishing, 2nd ed., 1979.

[24] A. Naor, J. Verstraëte, A note on bipartite graphs without $2 k$-cycles, Combinatorics, Probability, and Computing 14 (2005), p. 845-849.

[25] O. Pikhurko, A note on the Turán function of even cycles, Proc. Amer. Math. Soc., to appear.

[26] J. Verstraëte, Arithmetic progressions of cycle lengths in graphs, Combinatorics, Probability, and Computing 9 (2000), 369-373.

[27] R. Wenger, Extremal graphs with no $C^{4}$ 's, $C^{6}$ 's, or $C^{10}$ 's, J. of Combinatorial Theory Series B, 52 (1991), p. 113-116. 